\title{
Decoherence in an exactly solvable qubit model with initial qubit-environment correlations
}

\author{
V.G. Morozov* \\ Moscow State Technical University of Radioengineering, Electronics, \\ and Automation, Vernadsky Prospect 78, 119454 Moscow, Russia \\ S. Mathey $\dagger^{\dagger}$ and G. Röpk $\oplus^{\ddagger}$ \\ University of Rostock, FB Physik, Universitätsplatz 3, D-18051 Rostock, Germany
}

(Dated: August 23, 2021)

\begin{abstract}
We study a model of dephasing (decoherence) in a two-state quantum system (qubit) coupled to a bath of harmonic oscillators. An exact analytic solution for the reduced dynamics of a two-state system in this model has been obtained previously for factorizing initial states of the combined system. We show that the model admits exact solutions for a large class of correlated initial states which are typical in the theory of quantum measurements. We derive exact expressions for the off-diagonal elements of the qubit density matrix, which hold for an arbitrary strength of coupling between the qubit and the bath. The influence of initial correlations on decoherence is considered for different bath spectral densities. Time behavior of the qubit entropy in the decoherence process is discussed.
\end{abstract}

PACS numbers: 03.65.Ta, 03.65.Yz

\section{INTRODUCTION}

There are two long-standing problems in the theory of open quantum systems - memory effects and the influence of initial statistical correlations on the system dynamics 1, 2. These problems take on special significance in the theory of decoherence (i.e., the environmentinduced destruction of quantum coherence), since the decoherence time scale is usually much shorter than the time scales for other relaxation processes in a system. The well-known Nakajima-Zwanzig projection operator technique [3, 4, and its modifications [1] provide formally exact non-Markovian master equations for almost arbitrary open systems and initial conditions. Unfortunately, it is impossible to solve these equations for more or less realistic models. Thanks to the work of many people (see, e.g., Refs. 1, 5, 6] and references therein), we possess today some partial advancements in constructing reasonable approximations for non-Markovian dynamics of open quantum systems, but a systematic general approach is still lacking.

The simplest systems involving many of fundamental features of quantum coherence are two-state systems. Such systems are important in their own right as the elementary carriers of quantum information (qubits) [7 9 . In addition, some two-state models admit exact solutions. The latter fact is very useful because it allows one to gain valuable insight into general properties of the dynamics of decoherence and can serve as a step toward consistent approximations for more complicated open systems. For instance, in Ref. [5, 6] two exactly solvable models have

\footnotetext{
* vladmorozov45@gmail.com

$\dagger$ mathey@thphys.uni-heidelberg.de

$\ddagger$ gerd.roepke@uni-rostock.de
}

been used to reconstruct the corresponding exact nonMarkovian master equations and study in detail their memory kernels. It would be interesting to apply a similar approach to situations with non-negligible initial correlations between an open system and its environment.

Recently [10, an exact analytic solution was obtained for a qubit model 11-13 with initial qubit-environment correlations. The results demonstrate new interesting features of decoherence in the presence of initial correlations. Unfortunately, the initial state considered in Ref. [10] seems to be somewhat artificial since it relies on the assumption that the qubit + environment system is initially prepared in a pure quantum state at zero temperature. In this paper we show that the model [1113] admits exact solutions for a large class of physically reasonable correlated initial states at finite temperatures and derive explicit expressions for the coherences (offdiagonal elements of the qubit density matrix).

The distinctive feature of the dephasing model [11 13 is that the average populations of the qubit states do not depend on time. In other words, there is no relaxation to complete equilibrium between the qubit and the environment, i.e., the model is nonergodic. Curiously enough this feature may be considered as an advantage of the model. Indeed, since the relaxation time $\tau_{R}$ for dissipative processes is usually much larger than the decoherence (dephasing) time $\tau_{D}$, the model [11 13] corresponds to the limiting case $\tau_{R} / \tau_{D} \rightarrow \infty$, when all irrelevant corrections due to energy dissipation are removed. Thus one may expect that essential features of decoherence in this simple model will be similar to those in more involved but less tractable dissipative models.

The paper is structured as follows. In Sec. II we give a brief description of the model and find exact solutions of equations of motion for all relevant operators. In Sec. III we derive exact expressions for the elements of the qubit 
density matrix, which hold for correlated initial conditions. These expressions are used in Sec. IV to study possible regimes of decoherence for different bath spectral densities. The results are compared with the uncorrelated case. Finally, in Sec. V we derive an exact expression for the qubit entropy and discuss its time behavior in the presence of initial qubit-environment correlations.

\section{THE MODEL}

We consider a simple version of a spin-boson model describing a two-state system $(S)$ coupled to a bath $(B)$ of harmonic oscillators [1, 11 13]. In the "spin" representation for a qubit, the total Hamiltonian of the model is written as (in our units $\hbar=1$ )

$$
\begin{aligned}
H & =H_{S}+H_{B}+H_{\mathrm{int}} \\
& =\frac{\omega_{0}}{2} \sigma_{3}+\sum_{k} \omega_{k} b_{k}^{\dagger} b_{k}+\sigma_{3} \sum_{k}\left(g_{k} b_{k}^{\dagger}+g_{k}^{*} b_{k}\right),
\end{aligned}
$$

where $\omega_{0}$ is the energy difference between the excited state $|1\rangle$ and the ground state $|0\rangle$ of the qubit, and $\sigma_{3}$ is one of the Pauli matrices $\sigma_{1}, \sigma_{2}, \sigma_{3}$. Note that $\sigma_{3}|1\rangle=$ $|1\rangle$ and $\sigma_{3}|0\rangle=-|0\rangle$. Bosonic creation and annihilation operators $b_{k}^{\dagger}$ and $b_{k}$ correspond to the $k$ th bath mode with frequency $\omega_{k}$.

Suppose that at time $t=0$ the state of the total system is described by some initial density matrix $\varrho(0)$. Then at time $t$ the density matrix is given by

$$
\varrho(t)=\exp (-i H t) \varrho(0) \exp (i H t) .
$$

Our main interest is with the reduced density matrix of the qubit

$$
\varrho_{S}(t)=\operatorname{Tr}_{B} \varrho(t),
$$

where $\operatorname{Tr}_{B}$ denotes the trace taken over the bath degrees of freedom. It is easy to see that

$$
\begin{aligned}
& \left\langle 0\left|\varrho_{S}(t)\right| 0\right\rangle=\frac{1}{2}\left\{1-\left\langle\sigma_{3}(t)\right\rangle\right\}, \\
& \left\langle 1\left|\varrho_{S}(t)\right| 1\right\rangle=\frac{1}{2}\left\{1+\left\langle\sigma_{3}(t)\right\rangle\right\}, \\
& \left\langle 0\left|\varrho_{S}(t)\right| 1\right\rangle=\left\langle\sigma_{+}(t)\right\rangle, \quad\left\langle 1\left|\varrho_{S}(t)\right| 0\right\rangle=\left\langle\sigma_{-}(t)\right\rangle,
\end{aligned}
$$

where $\sigma_{ \pm}=\left(\sigma_{1} \pm i \sigma_{2}\right) / 2$. Here and in the following the symbol $\langle A(t)\rangle$ stands for the average value of a Heisenberg picture operator calculated with the initial density matrix of the total system:

$$
\langle A(t)\rangle=\operatorname{Tr}\{\exp (i H t) A \exp (-i H t) \varrho(0)\} .
$$

The notation $\langle A\rangle$ will be used for averages at $t=0$.

In the model (1), equations of motion for all relevant operators can be solved exactly. Technical details are summarized in Appendix A. Here we quote the results. The time-dependent bath operators are given by

$$
\begin{aligned}
& b_{k}(t)=\mathrm{e}^{-i \omega_{k} t}\left[b_{k}+\frac{\sigma_{3}}{2} \alpha_{k}(t)\right], \\
& b_{k}^{\dagger}(t)=\mathrm{e}^{i \omega_{k} t}\left[b_{k}^{\dagger}+\frac{\sigma_{3}}{2} \alpha_{k}^{*}(t)\right],
\end{aligned}
$$

with

$$
\alpha_{k}(t)=2 g_{k} \frac{1-\mathrm{e}^{i \omega_{k} t}}{\omega_{k}},
$$

and the qubit operators $\sigma_{ \pm}(t)$ can be written as

$$
\sigma_{ \pm}(t)=\exp \left[ \pm i \omega_{0} t \mp R(t)\right] \sigma_{ \pm},
$$

where the operator $R(t)$ acts only on the bath states:

$$
R(t)=\sum_{k}\left[\alpha_{k}(t) b_{k}^{\dagger}-\alpha_{k}^{*}(t) b_{k}\right] .
$$

Since $\sigma_{3}$ commutes with the Hamiltonian (1), we have $\sigma_{3}(t)=\sigma_{3}$, so that the populations $\left\langle 0\left|\varrho_{S}(t)\right| 0\right\rangle$ and $\left\langle 1\left|\varrho_{S}(t)\right| 1\right\rangle$ do not depend on time.

\section{EXACT SOLUTIONS FOR THE COHERENCES}

\section{A. Uncorrelated initial state}

As an introduction to our subsequent development, we first briefly review the calculation of the coherences $\left\langle\sigma_{ \pm}(t)\right\rangle$ in the case that the qubit and the bath are initially uncorrelated, and the bath is in thermal equilibrium at some temperature $T$ 1, 11,13. In this case the initial density matrix of the total system is a direct product

$$
\varrho(0)=\varrho_{S}(0) \otimes \varrho_{B}, \quad \varrho_{B}=\mathrm{e}^{-\beta H_{B}} / Z_{B},
$$

where $\beta=1 / k_{\mathrm{B}} T$, and $Z_{B}$ is the bath partition function. Note that $\varrho_{S}(0)$ may be a pure state as well as a mixed state of the qubit.

Using expressions (6) and (8), one obtains

$$
\left\langle\sigma_{ \pm}(t)\right\rangle=\left\langle\sigma_{ \pm}\right\rangle \mathrm{e}^{ \pm i \omega_{0} t} \mathrm{e}^{-\gamma(t)}
$$

with the decoherence function $\gamma(t)$ defined as

$$
\begin{aligned}
\gamma(t) & =-\ln \left\langle\mathrm{e}^{\mp R(t)}\right\rangle_{B} \\
& =-\sum_{k} \ln \left\langle\exp \left[\alpha_{k}(t) b_{k}^{\dagger}-\alpha_{k}^{*}(t) b_{k}\right]\right\rangle_{B},
\end{aligned}
$$

where the symbol $\langle\ldots\rangle_{B}$ denotes averages taken with the bath distribution $\varrho_{B}$. After straightforward algebra (see, e.g., Ref. [1]) one finds

$$
\gamma(t)=\int_{0}^{\infty} d \omega J(\omega) \operatorname{coth}(\beta \omega / 2) \frac{1-\cos \omega t}{\omega^{2}},
$$

were the continuum limit of the bath modes is performed, and the spectral density $J(\omega)$ is introduced by the rule

$$
\sum_{k} 4\left|g_{k}\right|^{2} f\left(\omega_{k}\right)=\int_{0}^{\infty} d \omega J(\omega) f(\omega) .
$$

Expression (11) is the exact result for the decoherence function in the model (1) under the uncorrelated initial condition 8. 


\section{B. Correlated initial states}

We turn now to the coherences $\left\langle\sigma_{ \pm}(t)\right\rangle$ for correlated initial states. First of all, we have to specify the initial density matrix $\varrho(0)$. Generally speaking, one may imagine a variety of different forms of this density matrix, but we will restrict our consideration to initial states

$$
\varrho(0)=\frac{1}{Z} \sum_{m} \Omega_{m} \mathrm{e}^{-\beta H} \Omega_{m}^{\dagger}
$$

where operators $\Omega_{m}$ act on the qubit states, and the partition function $Z$ ensures the normalization of $\varrho(0)$. Such density matrices are used, for instance, to describe the preparation of a system by means of a quantum measurement [1]. They also arise in a natural way in the calculation of correlation functions of open quantum systems. In the theory of decoherence, of special interest are initial density matrices [1, 2]

$$
\varrho(0)=\frac{1}{Z} P_{\psi} \mathrm{e}^{-\beta H} P_{\psi}
$$

where $P_{\psi}=|\psi\rangle\langle\psi|$ is the projector onto a pure quantum state $|\psi\rangle$. In the usual representation, we have

$$
|\psi\rangle=a_{0}|0\rangle+a_{1}|1\rangle
$$

with $\left|a_{0}\right|^{2}+\left|a_{1}\right|^{2}=1$. The projector $P_{\psi}$ can also be written as

$$
P_{\psi}=\frac{1}{2}(1+\vec{\sigma} \cdot \vec{p}), \quad|\vec{p}|=1,
$$

where the components of $\vec{p}$ are easily expressed in terms of the amplitudes $a_{0}$ and $a_{1}$. The density matrix (14) corresponds to the so-called selective quantum measurement [1] and is especially suited to study the environment-induced destruction of quantum coherence.

In contrast to density matrices of the form (8), the density matrices (13) and (14) contain the total Hamiltonian of the system and, consequently, describe initial qubitbath correlations. If we neglect the interaction term $H_{\text {int }}$, replacing $H$ by $H_{0}=H_{S}+H_{B}$, then we immediately recover an uncorrelated state (8). It is interesting to note that the density matrix 14 can be written as

$$
\varrho(0)=P_{\psi} \otimes \varrho_{B}(|\psi\rangle)
$$

where $\varrho_{B}(|\psi\rangle)$ plays the role of the initial density matrix of the bath and, at the same time, is a functional of $|\psi\rangle$ :

$$
\varrho_{B}(|\psi\rangle)=\frac{\langle\psi|\exp (-\beta H)| \psi\rangle}{\operatorname{Tr}_{B}\langle\psi|\exp (-\beta H)| \psi\rangle} .
$$

In the model under consideration, this functional can be obtained explicitly. First we note that the Hamiltonian (1) satisfies

where

$$
\begin{aligned}
& \mathrm{e}^{-\beta H}|0\rangle=\mathrm{e}^{\beta \omega_{0} / 2} \mathrm{e}^{-\beta H_{B}^{(-)}} \otimes|0\rangle, \\
& \mathrm{e}^{-\beta H}|1\rangle=\mathrm{e}^{-\beta \omega_{0} / 2} \mathrm{e}^{-\beta H_{B}^{(+)}} \otimes|1\rangle,
\end{aligned}
$$

$$
H_{B}^{( \pm)}=\sum_{k} \omega_{k} b_{k}^{\dagger} b_{k} \pm \sum_{k}\left(g_{k} b_{k}^{\dagger}+g_{k}^{*} b_{k}\right) .
$$

Then, with Eqs. 19, the initial bath density matrix 18 is manipulated to

$$
\varrho_{B}(|\psi\rangle)=\frac{\left|a_{0}\right|^{2} \mathrm{e}^{\beta \omega_{0} / 2} \mathrm{e}^{-\beta H_{B}^{(-)}}+\left|a_{1}\right|^{2} \mathrm{e}^{-\beta \omega_{0} / 2} \mathrm{e}^{-\beta H_{B}^{(+)}}}{\left|a_{0}\right|^{2} \mathrm{e}^{\beta \omega_{0} / 2} Z_{B}^{(-)}+\left|a_{1}\right|^{2} \mathrm{e}^{-\beta \omega_{0} / 2} Z_{B}^{(+)}},
$$

where

$$
Z_{B}^{( \pm)}=\operatorname{Tr}_{B} \exp \left[-\beta H_{B}^{( \pm)}\right]
$$

Thus, although the density matrices (8) and (17) are similar in form, the latter involves initial qubit-bath correlations. As shown below, these correlations can significantly affect the dynamics of the total system.

\section{Time evolution of correlated initial states}

The structure of the coherences $\left\langle\sigma_{ \pm}(t)\right\rangle$ can be investigated for arbitrary operators $\Omega_{m}$ in Eq. (13). First we write

$$
\begin{aligned}
\left\langle\sigma_{ \pm}(t)\right\rangle & =\frac{1}{Z} \sum_{m} \operatorname{Tr}\left[\Omega_{m}^{\dagger} \sigma_{ \pm}(t) \Omega_{m} \mathrm{e}^{-\beta H}\right] \\
& =\frac{\mathrm{e}^{ \pm i \omega_{0} t}}{Z} \sum_{m} \operatorname{Tr}_{B}\left\{\operatorname{Tr}_{S}\left[\Omega_{m}^{\dagger} \sigma_{ \pm} \Omega_{m} \mathrm{e}^{\mp R(t)} \mathrm{e}^{-\beta H}\right]\right\} .
\end{aligned}
$$

Using Eqs. 19, the above expression is recast into

$$
\begin{aligned}
\left\langle\sigma_{ \pm}(t)\right\rangle=\frac{\mathrm{e}^{ \pm i \omega_{0} t}}{Z} \sum_{m}\left\{\mathrm{e}^{\beta \omega_{0} / 2}\left\langle 0\left|\Omega_{m}^{\dagger} \sigma_{ \pm} \Omega_{m}\right| 0\right\rangle \operatorname{Tr}_{B}\left(\mathrm{e}^{\mp R(t)} \mathrm{e}^{-\beta H_{B}^{(-)}}\right)\right. & \\
& \left.+\mathrm{e}^{-\beta \omega_{0} / 2}\left\langle 1\left|\Omega_{m}^{\dagger} \sigma_{ \pm} \Omega_{m}\right| 1\right\rangle \operatorname{Tr}_{B}\left(\mathrm{e}^{\mp R(t)} \mathrm{e}^{-\beta H_{B}^{(+)}}\right)\right\}
\end{aligned}
$$


with the partition function

$$
\begin{aligned}
Z= & \sum_{m}\left\{\mathrm{e}^{\beta \omega_{0} / 2}\left\langle 0\left|\Omega_{m}^{\dagger} \Omega_{m}\right| 0\right\rangle Z_{B}^{(-)}\right. \\
& \left.+\mathrm{e}^{-\beta \omega_{0} / 2}\left\langle 1\left|\Omega_{m}^{\dagger} \Omega_{m}\right| 1\right\rangle Z_{B}^{(+)}\right\} .
\end{aligned}
$$

We now note that the traces $\operatorname{Tr}_{B}(\ldots)$ in Eq. 23) can be simplified considerably by a unitary transformation of $H_{B}^{( \pm)}$and $R(t)$ with

$$
U_{ \pm}=\exp \left\{ \pm \sum_{k}\left(\frac{g_{k}}{\omega_{k}} b_{k}^{\dagger}-\frac{g_{k}^{*}}{\omega_{k}} b_{k}\right)\right\}
$$

It is easy to verify that

$$
\begin{aligned}
& U_{ \pm} H_{B}^{( \pm)} U_{ \pm}^{-1}=H_{B}-\sum_{k} \frac{\left|g_{k}\right|^{2}}{\omega_{k}}, \\
& U_{ \pm} R(t) U_{ \pm}^{-1}=R(t) \pm i \Phi(t),
\end{aligned}
$$

where we have introduced the $c$-number function

$$
\Phi(t)=\sum_{k} \frac{4\left|g_{k}\right|^{2}}{\omega_{k}^{2}} \sin \left(\omega_{k} t\right)=\int_{0}^{\infty} d \omega J(\omega) \frac{\sin \omega t}{\omega^{2}}
$$

with the same spectral density $J(\omega)$ as in Eq. (11). Using the transformation properties (25) in Eqs. 23) and (24), we obtain the final expression for the coherences, which can be conveniently written as

$$
\left\langle\sigma_{ \pm}(t)\right\rangle=\left\langle\sigma_{ \pm}\right\rangle \mathrm{e}^{ \pm i \omega_{0} t} \mathrm{e}^{-\gamma(t)} \frac{\sum_{m}\left\{\left\langle 0\left|\Omega_{m}^{\dagger} \sigma_{ \pm} \Omega_{m}\right| 0\right\rangle \mathrm{e}^{\beta \omega_{0} / 2} \mathrm{e}^{ \pm i \Phi(t)}+\left\langle 1\left|\Omega_{m}^{\dagger} \sigma_{ \pm} \Omega_{m}\right| 1\right\rangle \mathrm{e}^{-\beta \omega_{0} / 2} \mathrm{e}^{\mp i \Phi(t)}\right\}}{\sum_{m}\left\{\left\langle 0\left|\Omega_{m}^{\dagger} \sigma_{ \pm} \Omega_{m}\right| 0\right\rangle \mathrm{e}^{\beta \omega_{0} / 2}+\left\langle 1\left|\Omega_{m}^{\dagger} \sigma_{ \pm} \Omega_{m}\right| 1\right\rangle \mathrm{e}^{-\beta \omega_{0} / 2}\right\}},
$$

where $\left\langle\sigma_{ \pm}\right\rangle$are initial values of the coherences.

Formulas 26 and 27 give an exact result for the decoherences in the model (1) with initial correlated states of the form (13). Expression (27) is considerably simplified in the special case when the initial density matrix is given by Eq. (14). After some algebra which we omit, we obtain

$$
\begin{aligned}
& \left\langle\sigma_{ \pm}(t)\right\rangle=\left\langle\sigma_{ \pm}\right\rangle \mathrm{e}^{ \pm i \omega_{0} t} \mathrm{e}^{-\gamma(t)}\{\cos [\Phi(t)] \\
& \left.\quad \pm i \frac{\sinh \left(\beta \omega_{0} / 2\right)-\left\langle\sigma_{3}\right\rangle \cosh \left(\beta \omega_{0} / 2\right)}{\cosh \left(\beta \omega_{0} / 2\right)-\left\langle\sigma_{3}\right\rangle \sinh \left(\beta \omega_{0} / 2\right)} \sin [\Phi(t)]\right\}
\end{aligned}
$$

where $\left\langle\sigma_{3}\right\rangle=\left|a_{1}\right|^{2}-\left|a_{0}\right|^{2}$. This expression can be represented in a more transparent form

$$
\left\langle\sigma_{ \pm}(t)\right\rangle=\left\langle\sigma_{ \pm}\right\rangle \mathrm{e}^{ \pm i\left[\omega_{0} t+\chi(t)\right]} \mathrm{e}^{-\widetilde{\gamma}(t)},
$$

where

$$
\widetilde{\gamma}(t)=\gamma(t)+\gamma_{\text {corr }}(t)
$$

is the modified decoherence function which includes the correlation contribution

$$
\begin{aligned}
& \gamma_{\text {corr }}(t) \\
& =-\frac{1}{2} \ln \left[1-\frac{\left(1-\left\langle\sigma_{3}\right\rangle^{2}\right) \sin ^{2}[\Phi(t)]}{\left[\cosh \left(\beta \omega_{0} / 2\right)-\left\langle\sigma_{3}\right\rangle \sinh \left(\beta \omega_{0} / 2\right)\right]^{2}}\right],
\end{aligned}
$$

and $\chi(t)$ is the time-dependent phase shift with

$$
\tan [\chi(t)]=\frac{\sinh \left(\beta \omega_{0} / 2\right)-\left\langle\sigma_{3}\right\rangle \cosh \left(\beta \omega_{0} / 2\right)}{\cosh \left(\beta \omega_{0} / 2\right)-\left\langle\sigma_{3}\right\rangle \sinh \left(\beta \omega_{0} / 2\right)} \tan [\Phi(t)]
$$

It is a straightforward matter to derive a formula analogous to Eq. (29) for more general initial states $(13)$ because each of the operators $\Omega_{m}$ in Eq. 27) can always be represented as $\Omega_{m}=c_{m}+\vec{\sigma} \cdot \vec{p}_{m}$ with some (in general, complex) $c_{m}$ and $\vec{p}_{m}$. The resulting expressions for $\gamma_{\text {corr }}(t)$ and $\chi(t)$ are rather cumbersome and will not be given here. From now on we restrict our discussion to Eqs. $29-(32)$.

\section{REGIMES OF DECOHERENCE FOR DIFFERENT BATH SPECTRAL DENSITIES}

As already noted, the model (1) is nonergodic, i.e., it does not describe the establishment of complete thermal equilibrium between the qubit and the bath since $\left\langle\sigma_{3}(t)\right\rangle=$ const. In this connection it is of interest to investigate the long-time behavior of the decoherence function (30). Suppose, for instance, that $\widetilde{\gamma}(t)$ is a bounded function. Then, in the limit as $t \rightarrow \infty$, the averages $\left\langle\sigma_{ \pm}(t)\right\rangle$ do not tend to zero, so that the destruction of quantum coherence is "incomplete". Physically, in this case the final stage of decoherence is determined by slow processes involving exchange of energy between the qubit and the environment, which are not included into the Hamiltonian (1). On the other hand, if $\widetilde{\gamma}(t) \rightarrow \infty$ as $t \rightarrow \infty$, then $\left\langle\sigma_{ \pm}(t)\right\rangle \rightarrow 0$ showing complete decoherence within the framework of the model under consideration.

Note that the correlation corrections in Eq. (31) [or, for a more general case, in Eq. [27]] are always bounded. This means that initial correlations alone cannot lead to complete decoherence. Let us now turn to the "dynamical" part of the decoherence function given by Eq. (11). It can be written conveniently as a sum

$$
\gamma(t)=\gamma_{\mathrm{vac}}(t)+\gamma_{\mathrm{th}}(t)
$$


where

$$
\gamma_{\mathrm{vac}}(t)=\int_{0}^{\infty} d \omega J(\omega) \frac{1-\cos \omega t}{\omega^{2}}
$$

is the contribution to the decoherence function from vacuum fluctuations in the bath, and

$$
\begin{aligned}
\gamma_{\mathrm{th}}(t) & =\int_{0}^{\infty} d \omega J(\omega)[\operatorname{coth}(\beta \omega / 2)-1] \frac{1-\cos \omega t}{\omega^{2}} \\
& =2 \int_{0}^{\infty} d \omega \frac{J(\omega)}{\mathrm{e}^{\beta \omega}-1} \frac{1-\cos \omega t}{\omega^{2}}
\end{aligned}
$$

is the temperature dependent thermal contribution. We have obvious inequalities

$$
\begin{aligned}
& \gamma_{\mathrm{vac}}(t) \leq 2 \int_{0}^{\infty} d \omega \frac{J(\omega)}{\omega^{2}}, \\
& \gamma_{\mathrm{th}}(t) \leq 4 \int_{0}^{\infty} d \omega \frac{J(\omega)}{\omega^{2}\left(\mathrm{e}^{\beta \omega}-1\right)},
\end{aligned}
$$

which show that $\gamma_{\mathrm{vac}}(t)$ and $\gamma_{\mathrm{th}}(t)$ are bounded if the integrals converge.

Another important question is whether, and under what conditions, the total decoherence function $\widetilde{\gamma}(t)$ tends to a definite limit as $t \rightarrow \infty$. Necessary conditions for different terms in Eq. (30) to have definite long-time limits can be derived by using relation [14]

$$
\lim _{t \rightarrow \infty} f(t)=\lim _{\varepsilon \rightarrow+0} \varepsilon \int_{0}^{\infty} d t \mathrm{e}^{-\varepsilon t} f(t)
$$

which is valid if the limit on the left-hand side exists. When applied to functions (34), 35), and (26), Eq. (37) gives

$$
\begin{aligned}
& \lim _{t \rightarrow \infty} \gamma_{\mathrm{vac}}(t)=\int_{0}^{\infty} d \omega \frac{J(\omega)}{\omega^{2}}, \\
& \lim _{t \rightarrow \infty} \gamma_{\mathrm{th}}(t)=2 \int_{0}^{\infty} d \omega \frac{J(\omega)}{\omega^{2}\left(\mathrm{e}^{\beta \omega}-1\right)}, \\
& \lim _{t \rightarrow \infty} \Phi(t)=\frac{\pi}{2} \lim _{\omega \rightarrow 0} \frac{J(\omega)}{\omega} .
\end{aligned}
$$

To go beyond relations (36) and (38), one needs some information about the bath spectral density. Generally speaking, its form can be obtained from a fully macroscopic analysis of the system-bath interactions leading to the spin-boson model (1). It would be beyond the scope of this paper to present a detailed discussion of such derivations. In many cases of practical interest (see, e.g., Refs. [13, 15]), $J(\omega)$ may be considered to be a reasonably smooth function which has a power-law behavior $J(\omega) \propto \omega^{s}(s>0)$ at frequencies much less than some "cutoff" frequency $\Omega$, characteristic of the bath modes. In the limit $\omega \rightarrow \infty, J(\omega)$ is assumed to fall off at least as some negative power of $\omega$. Then, on dimensional grounds, we may write

$$
J(\omega)=\lambda_{s} \Omega^{1-s} \omega^{s} F(\omega / \Omega),
$$

where $\lambda_{s}$ is a dimensionless coupling constant, and a cutoff function satisfies

$$
F(0)=1, \quad \lim _{\omega / \Omega \rightarrow \infty} F(\omega / \Omega)=0 .
$$

The case $s=1$ is usually called the "ohmic" case, the case $s>1$ "superohmic", and the case $0<s<1$ "subohmic".

As is easy to see, the convergence of the integrals in Eqs. (36) and (38) depends crucially on the low-frequency behavior of $J(\omega)$. Assuming a power law for $J(\omega)$ in this region, we can make some conclusions about possible regimes of decoherence in the model (1). Let us first of all note that both integrals in inequalities (36) converge for $s>2$. Therefore, in this case the total decoherence function $\widetilde{\gamma}(t)$ is bounded, so that the above mentioned regime of "incomplete decoherence" takes place. It is also seen from Eqs. (36) that, in the superohmic case with $1<s \leq 2$, the thermal term $\gamma_{\text {th }}(t)$ is the only contribution to the total decoherence function which may diverge as $t \rightarrow \infty$. Finally, Eq. (38c) shows that the correlation term $\gamma_{\text {corr }}(t)$ may have a nonzero long-time limit only in the ohmic case $(s=1)$.

The virtue of the above conclusions is that they apply to all spectral densities of the form (39), but they say nothing about the regime of decoherence for $0<s \leq 2$. It is reasonable to expect that in this range of values of the parameter $s$ we are dealing with the regime of "complete decoherence", since the vacuum term $\gamma_{\text {vac }}(t)$ may diverge as $t \rightarrow \infty$ for $0<s \leq 1$ and the thermal term $\gamma_{\text {th }}(t)$ for $0<s \leq 2$. We could not determine, however, a general sufficient condition for the cutoff function in Eq. (39) to ensure that $\gamma_{\mathrm{vac}}(t)$ and $\gamma_{\mathrm{th}}(t)$ behave in this manner. Note, however, that this question is largely academic since the model specified by the Hamiltonian (1), on its own, is of most physical interest in the lowtemperature range $(\Omega \beta \gg 1)$ where dissipative processes are irrelevant and the bath dynamics is completely determined by low-frequency modes with $\omega_{k} \ll \Omega$. For this temperature range, we show in Appendix B that, under assumptions (39) and (40), the thermal term $\gamma_{\text {th }}(t)$ is a steadily increasing function of time for $0<s \leq 2$, and hence $\gamma_{\mathrm{th}}(t) \rightarrow \infty$ as $t \rightarrow \infty$. This means that the regime of "complete decoherence" takes place for all $0<s \leq 2$.

Up to this point we have been concerned with those features of decoherence which do not depend on the form of the bath spectral density or, in particular, on the form of the cutoff function in Eq. (39). For specific cases, functions (31), (34), and (35) can be evaluated numerically. However, to gain more insight into the overall picture of decoherence, it will be instructive to consider a particular choice of the cutoff function in Eq. (39) for which all the quantities of interest can be evaluated exactly. We shall take for the bath spectral density the expression

$$
J(\omega)=\lambda_{s} \Omega^{1-s} \omega^{s} \mathrm{e}^{-\omega / \Omega},
$$

which is most commonly used in the theory of spin-boson systems [1, 11, 13, 15]. 
Let us start with the vacuum term (34) in the decoherence function. Substituting here the spectral density from Eq. (41) and doing standard integrals, we get

$$
\begin{aligned}
& \gamma_{\mathrm{vac}}(t)=\lambda_{s} \Gamma(s-1) \\
& \times\left\{1-\frac{\cos \left[(s-1) \tan ^{-1}(\Omega t)\right]}{\left(1+\Omega^{2} t^{2}\right)^{(s-1) / 2}}\right\}, \quad(s \neq 1), \\
& \gamma_{\text {vac }}(t)=\frac{\lambda_{1}}{2} \ln \left(1+\Omega^{2} t^{2}\right), \quad(s=1) .
\end{aligned}
$$

The latter expression is identical with the well known result [1. Note that the properties of $\gamma_{\mathrm{vac}}(t)$ given by Eqs. 42 are consistent with the general statements formulated in the beginning of this section. First, the vacuum term is a monotonically increasing function of time for $s \leq 1$. Second, in the superohmic case $(s>1)$ this term has a long-time limit:

$$
\lim _{t \rightarrow \infty} \gamma_{\mathrm{vac}}(t)=\lambda_{s} \Gamma(s-1), \quad(s>1)
$$

It is easy to check that this limit is exactly the same as given by Eq. (38a). One can also see from Eq. (42a) that $\gamma_{\text {vac }}(t)$ monotonically saturates to $\gamma_{\text {vac }}(\infty)$ for $1<s \leq 2$ and is a nonmonotonic function of time for $s>2$. These properties of the vacuum term are illustrated in Fig. 1 .

Evaluation of the thermal term 35 with the spectral density 41) also reduces to performing standard integrals. After some manipulations which we omit, we obtain

$$
\begin{aligned}
\gamma_{\mathrm{th}}(t)= & 2 \lambda_{s}(\Omega \beta)^{1-s} \Gamma(s-1) \\
& \times \sum_{k=1}^{\infty} \frac{1}{(k+1 / \Omega \beta)^{s-1}}\left\{1-\left[1+\frac{(t / \beta)^{2}}{(k+1 / \Omega \beta)^{2}}\right]^{-(s-1) / 2} \cos \left[(s-1) \varphi_{k}(t)\right]\right\}, \quad(s>0, s \neq 1), \\
\gamma_{\text {th }}(t)= & 2 \lambda_{1}\left[\ln \Gamma(1+1 / \Omega \beta)-\frac{1}{2} \ln |\Gamma(1+1 / \Omega \beta+i t / \beta)|^{2}\right], \quad(s=1),
\end{aligned}
$$

where we have introduced the notation

$$
\varphi_{k}(t)=\tan ^{-1}\left(\frac{t / \beta}{k+1 / \Omega \beta}\right) .
$$

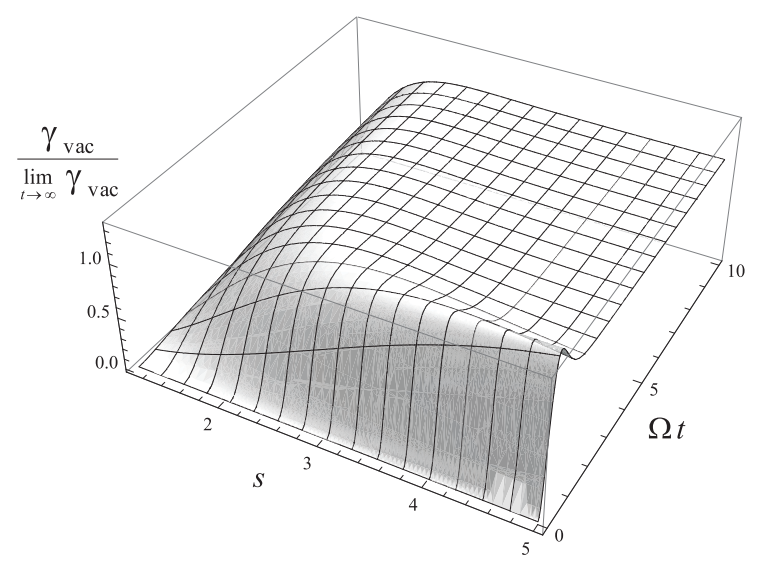

FIG. 1. Time dependence of the vacuum contribution to the decoherence function in the superohmic case $(s>1)$.

At low temperatures $(\Omega \beta \gg 1)$ expression $44 \mathrm{~b}$ reduces to the well known result [1, 12, 13.

$$
\begin{aligned}
\gamma_{\mathrm{th}}(t) & =-\lambda_{1} \ln |\Gamma(1+i t / \beta)|^{2} \\
& =\lambda_{1} \ln \left[\frac{\sinh \left(t / \tau_{B}\right)}{t / \tau_{B}}\right], \quad(s=1, \Omega \beta \gg 1),
\end{aligned}
$$

where

$$
\tau_{B}=\beta / \pi \equiv \hbar / \pi k_{\mathrm{B}} T
$$

is the so-called thermal correlation time.

We see from Eq. 44a that in the case of incomplete decoherence $(s>2)$ the thermal term has a long-time limit

$$
\begin{aligned}
\lim _{t \rightarrow \infty} \gamma_{\mathrm{th}}(t) & =2 \lambda_{s}(\Omega \beta)^{1-s} \\
\times & \Gamma(s-1) \zeta(s-1,1+1 / \Omega \beta), \quad(s>2),
\end{aligned}
$$

where $\zeta(z, v)$ is the generalized Riemann zeta function. One may easily verify that the above result is exactly the same as predicted by Eq. (38b). It is somewhat more difficult to clarify the time behavior of $\gamma_{\mathrm{th}}(t)$ directly from Eq. (44). We show in Appendix B that, for $0<s \leq 2$, $\gamma_{\mathrm{th}}(t)$ is a monotonically increasing function of time and $\gamma_{\text {th }}(t) \rightarrow \infty$ as $t \rightarrow \infty$. In the regime of "incomplete decoherence" $(s>2)$, the thermal term exhibits nonmonotonic time behavior for sufficiently large values of the parameter $s$ (see Appendix B and Fig. 2).

We now turn to the correlation term (31) in the decoherence function assuming the bath spectral density to be given by Eq. (41). As a preliminary step, we calculate 


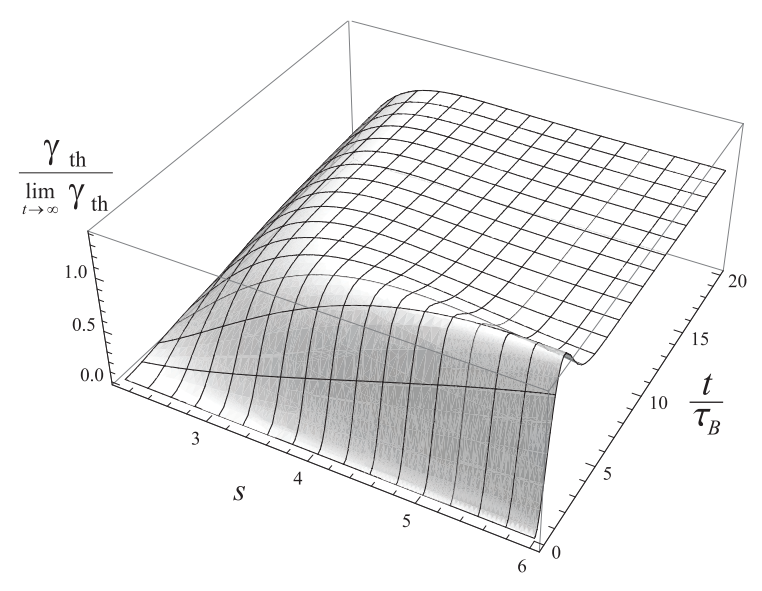

FIG. 2. Time dependence of the thermal contribution to the decoherence function in the regime of "incomplete decoherence" $(s>2) ; \tau_{B}$ is given by Eq. (47) and $\Omega \tau_{B}=10$.

the phase function (26). Doing the $\omega$-integral, we find

$$
\begin{aligned}
\Phi(t) & =\frac{\lambda_{s} \Gamma(s-1)}{\left(1+\Omega^{2} t^{2}\right)^{(s-1) / 2}} \\
& \times \sin \left[(s-1) \tan ^{-1}(\Omega t)\right], \quad(s>0, s \neq 1), \\
\Phi(t) & =\lambda_{1} \tan ^{-1}(\Omega t), \quad(s=1) .
\end{aligned}
$$

These expressions allow one to bring out some important properties of the correlation contribution to the decoherence function. First, in the subohmic case $(0<s<1)$, the phase function $49 \mathrm{a}$ increases with time and, consequently, $\gamma_{\text {corr }}(t)$ oscillates. Second, for $s \geq 1$ the phase function has long-time limits

$$
\begin{aligned}
& \lim _{t \rightarrow \infty} \Phi(t)=\lambda_{1} \pi / 2, \quad(s=1), \\
& \lim _{t \rightarrow \infty} \Phi(t)=0, \quad(s>1),
\end{aligned}
$$

which are consistent with Eq. (38c). We thus conclude that in the superohmic case $(s>1)$ the correlation term (31) asymptotically tends to zero as $t \rightarrow \infty$, whereas in the ohmic case $(s=1)$ it has a long-time limit

$$
\begin{aligned}
& \lim _{t \rightarrow \infty} \gamma_{\text {corr }}(t) \\
& -\frac{1}{2} \ln \left[1-\frac{\left(1-\left\langle\sigma_{3}\right\rangle^{2}\right) \sin ^{2}\left(\lambda_{1} \pi / 2\right)}{\left[\cosh \left(\beta \omega_{0} / 2\right)-\left\langle\sigma_{3}\right\rangle \sinh \left(\beta \omega_{0} / 2\right)\right]^{2}}\right] .
\end{aligned}
$$

It is interesting to note that this limiting value is a periodic function of the coupling constant $\lambda_{1}$. In particular, for $\lambda_{1}=2 n,(n=1,2, \ldots)$, we have $\gamma_{\text {corr }}(\infty)=0$. The time behavior of $\gamma_{\text {corr }}(t)$ in the ohmic case is very sensitive to the value of the coupling constant. For sufficiently large $\lambda_{1}, \gamma_{\text {corr }}(t)$ has a "peak" structure (see Fig. 3).

Qualitative properties of the contributions to the total decoherence function $\widetilde{\gamma}(t)$ for different values of the parameter $s$ in Eq. (41) are summarized in Table I. We note

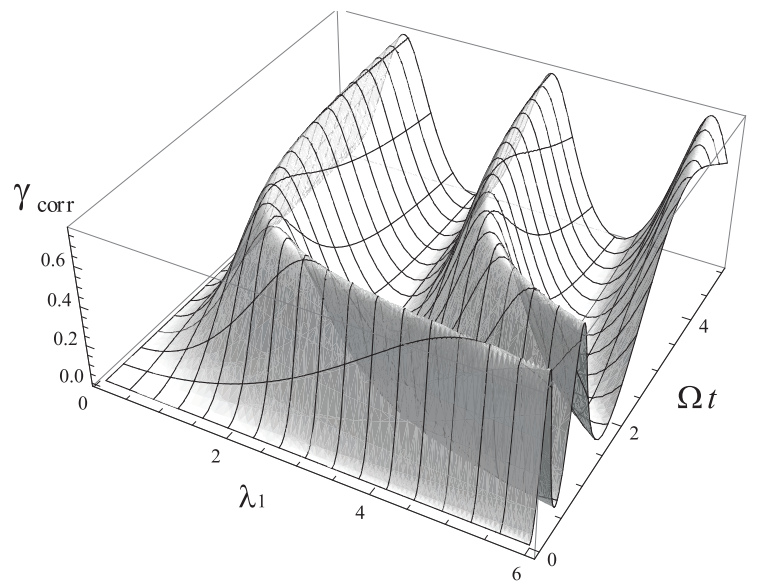

FIG. 3. Time dependence of the correlation contribution to the decoherence function in the ohmic case $(s=1)$ for different values of the coupling constant $\lambda_{1}$. Parameter values: $\left\langle\sigma_{3}\right\rangle=0$ (equal populations of the qubit states $|0\rangle$ and $|1\rangle$ ); $\omega_{0} \beta=1$.

that the value $s=2$ plays a role of a "critical parameter" for decoherence in the model (1). If $s \leq 2$, we have the regime of "complete decoherence" since $\widetilde{\gamma}(t) \rightarrow \infty$ as $t \rightarrow \infty$, and hence the coherences (29) asymptotically tend to zero. For $s>2$, the total decoherence function has a finite long-time limit determined by the vacuum and thermal contributions. In this case we are dealing with the regime of "incomplete decoherence". It should, however, be pointed out that in a real qubit the residual coherences $\left\langle\sigma_{ \pm}(\infty)\right\rangle$ decay to zero due to dissipative processes which are not included in the model (1).

We close this section with remarks about the role of initial qubit-bath correlations in different regimes of decoherence. Some conclusions concerning correlation effects can be drawn directly from Eq. (31). First, in the weak coupling limit $\left(\lambda_{s} \ll 1\right)$ Eqs. 490 and 31 give $\gamma_{\text {corr }} \propto \lambda_{s}^{2}$, while $\gamma_{\text {vac }} \propto \lambda_{s}$ and $\gamma_{\text {th }} \propto \lambda_{s}$. We see that in this limit the main contribution to the total decoherence function (30) is from its "dynamical" part $\gamma(t)$. Second, it is clear that the correlation term (31) is small compared to $\gamma(t)$ at extremely low temperatures $\left(\beta \omega_{0} \gg 1\right)$ for all values of $\lambda_{s}$. We thus conclude that the role of initial qubit-bath correlations becomes pronounced in the temperature region $\beta \omega_{0} \lesssim 1$ for intermediate strength of coupling.

Formulas 49 show that $\Omega^{-1}$ determines the characteristic time scale for the correlation effects. The same is true for the vacuum term (42). On the other hand, the quantity $\tau_{B}$ given by Eq. (47) determines the time scale for thermal effects in decoherence [cf. Eqs. (44) and (45)]. In the most interesting low-temperature range $(\Omega \beta \gg 1)$, we have $\Omega \tau_{B} \gg 1$, so that initial correlations might be expected to have a pronounced effect on the coherences $\left\langle\sigma_{ \pm}(t)\right\rangle$ at times $t<\tau_{B}$ when the main contribution to the "dynamical part" $\gamma(t)$ of the decoherence function 
TABLE I. Time behavior of different contributions to the total decoherence function $\widetilde{\gamma}(t)$ for the bath spectral density (41).

\begin{tabular}{cccc}
\hline \hline & $\gamma_{\text {vac }}(t)$ & $\gamma_{\text {th }}(t)$ & $\gamma_{\text {corr }}(t)$ \\
\hline $0<s<1$ & Monotonic increase & Monotonic increase & Oscillations \\
$s=1$ & Monotonic increase & Monotonic increase & "Peak" structure; \\
& & & $\begin{array}{l}\gamma_{\text {corr }}(\infty)=0 \text { or } \gamma_{\text {corr }}(\infty) \neq 0 \\
\text { depending on the value of } \lambda_{1}\end{array}$ \\
$1<s \leq 2$ & Saturation to $\gamma_{\text {vac }}(\infty) \neq 0$ & Monotonic increase & Nonmonotonic decay \\
$s>2$ & Saturation to $\gamma_{\text {vac }}(\infty) \neq 0$ & Saturation to $\gamma_{\text {th }}(\infty) \neq 0$ & Nonmonotonic decay \\
\hline \hline
\end{tabular}

comes from vacuum fluctuations in the bath. For the superohmic case, this can be clearly seen in Fig. 4 which illustrates the time behavior of the coherences in different regimes. Note, however, that in the subohmic and ohmic cases, for sufficiently weak coupling, the correlation effects manifest themselves at times $t>\tau_{B}$ where thermal excitations dominate (see Fig. 4). The reason can be traced to the facts that, in the former case, the correlation term $\gamma_{\text {corr }}(t)$ exhibits undamped oscillations, while in the latter case it has a long-time "plateau".

\section{ENTROPY}

Entropy plays a crucial role in the theory of open systems since it is a natural measure of the lack of information about a system. It is thus of interest to discuss the time behavior of the qubit entropy for the model under consideration.

We start from the general expression for the von Neumann (information) entropy of a quantum system:

$$
S(t)=-\operatorname{Tr}_{S}\left\{\varrho_{S}(t) \ln \varrho_{S}(t)\right\}
$$

In the case of a qubit, it is convenient to express $S(t)$ in terms of the Bloch vector $\vec{v}(t)=\langle\vec{\sigma}(t)\rangle$ using two representations for the density matrix $\varrho_{S}(t)$. The standard representation is [16, 17]

$$
\varrho_{S}(t)=\frac{1}{2}[1+\vec{\sigma} \cdot \vec{v}(t)]
$$

In Appendix $\mathrm{C}$ we derive another representation which is better suited to calculate $\ln \varrho_{S}(t)$ :

$$
\varrho_{S}(t)=\frac{1}{2} \sqrt{1-v^{2}(t)} \exp [\vec{\sigma} \cdot \vec{u}(t)]
$$

where $\vec{u} \uparrow \uparrow \vec{v}$, and

$$
u=\frac{1}{2} \ln \left(\frac{1+v}{1-v}\right) .
$$

Strictly speaking, formula (54) is valid only if $v<1$, i.e., for a mixed state. Note, however, that the limit $v \rightarrow 1$ can be taken directly in the entropy (52) after calculating the trace. With expressions (54) and 55 one easily derives from Eq. 52

$$
S(t)=\ln 2-\frac{1}{2}(1+v) \ln (1+v)-\frac{1}{2}(1-v) \ln (1-v) .
$$

For a pure state $(v \rightarrow 1)$ this formula gives $S=0$, as it should be.

The square modulus of the Bloch vector can in general be written as

$$
v^{2}(t)=4\left\langle\sigma_{+}(t)\right\rangle\left\langle\sigma_{-}(t)\right\rangle+\left\langle\sigma_{3}(t)\right\rangle^{2}
$$

For simplicity we shall restrict further discussion to correlated initial states of the form (14). Then we have $v(0)=1$ and, consequently,

$$
\left\langle\sigma_{+}\right\rangle\left\langle\sigma_{-}\right\rangle=\frac{1}{4}\left(1-\left\langle\sigma_{3}\right\rangle^{2}\right) \text {. }
$$

Now using the solution 29 and taking into account that $\sigma_{3}$ is an integral of motion, we obtain from Eq. (57)

$$
v(t)=\left[\left\langle\sigma_{3}\right\rangle^{2}+\left(1-\left\langle\sigma_{3}\right\rangle^{2}\right) \mathrm{e}^{-2 \widetilde{\gamma}(t)}\right]^{1 / 2} .
$$

Formulas 56 and (58) determine the time evolution of the qubit entropy.

In discussing the properties of entropy in the model (1) under the assumption (39) for the bath spectral density, it is necessary to distinguish two cases: the regime of "complete decoherence", and the regime of "incomplete decoherence". In the former case $(s \leq 2)$ we have $\widetilde{\gamma}(t) \rightarrow$ $\infty$ as $t \rightarrow \infty$, and hence the limiting value of the entropy is the same for both (correlated and uncorrelated) initial conditions:

$$
\begin{aligned}
\lim _{t \rightarrow \infty} S(t) & =\ln 2-\frac{1}{2}\left(1+\left|\left\langle\sigma_{3}\right\rangle\right|\right) \ln \left(1+\left|\left\langle\sigma_{3}\right\rangle\right|\right) \\
- & \frac{1}{2}\left(1-\left|\left\langle\sigma_{3}\right\rangle\right|\right) \ln \left(1-\left|\left\langle\sigma_{3}\right\rangle\right|\right), \quad(s \leq 2) .
\end{aligned}
$$

The maximum entropy $S_{\max }(\infty)=\ln 2$ corresponds to the initial state with equal populations $\left(\left\langle\sigma_{3}\right\rangle=0\right)$. A similar situation occurs in the case of "incomplete decoherence" $(s>2)$ since $\gamma_{\text {corr }}(t) \rightarrow 0$ as $t \rightarrow \infty$. Note, however, that the limiting value of the qubit entropy is now given by

$$
\begin{array}{r}
\lim _{t \rightarrow \infty} S(t)=\ln 2-\frac{1}{2}\left(1+v_{\infty}\right) \ln \left(1+v_{\infty}\right) \\
-\frac{1}{2}\left(1-v_{\infty}\right) \ln \left(1-v_{\infty}\right), \quad(s>2),
\end{array}
$$



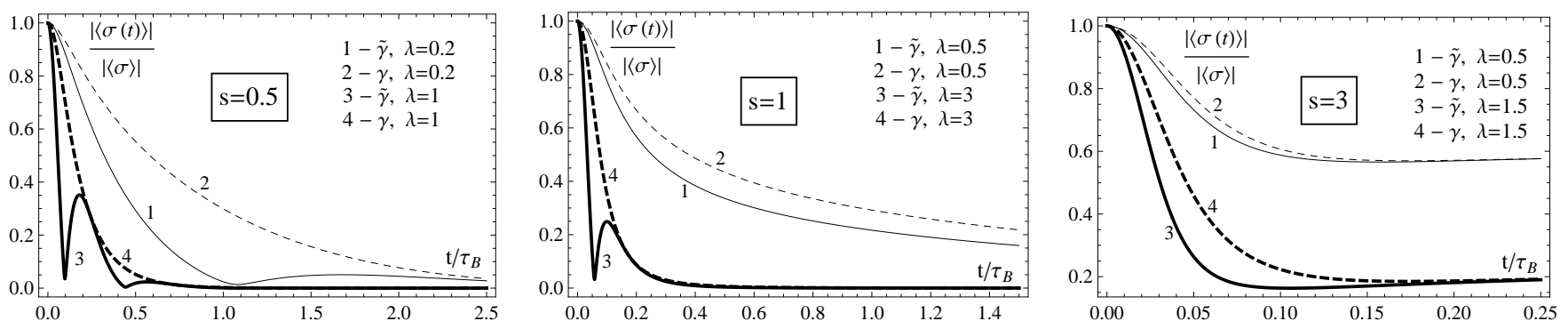

FIG. 4. Time dependence of the coherences $\langle\sigma(t)\rangle \equiv\left\langle\sigma_{ \pm}(t)\right\rangle$ for the correlated initial condition (14) (solid lines) and the uncorrelated initial condition (8) (dashed lines). In all cases $\lambda \equiv \lambda_{s} ; \tau_{B}$ is given by Eq. (47). Parameter values: $\Omega \tau_{B}=10$, $\omega_{0} \beta=0.1$.

where

$$
v_{\infty}=\left[\left\langle\sigma_{3}\right\rangle^{2}+\left(1-\left\langle\sigma_{3}\right\rangle^{2}\right) \mathrm{e}^{-2 \gamma(\infty)}\right]^{1 / 2} .
$$

Recalling Eqs. 437 and (48), we have

$$
\begin{aligned}
& \gamma(\infty)=\lambda_{s} \Gamma(s-1) \\
& \quad \times\left[1+\frac{2}{(\Omega \beta)^{s-1}} \zeta(s-1,1+1 / \Omega \beta)\right], \quad(s>2) .
\end{aligned}
$$

The second term in braces corresponds to the contribution from thermal excitations in the bath and is relatively small in the low-temperature range $(\Omega \beta \gg 1)$.

Although initial qubit-bath correlations do not contribute to $S(\infty)$ for all $s>0$, they influence the behavior of the qubit entropy at times $t<\tau_{B}$. The main reason is easy to see when one recalls that, for sufficiently strong coupling, the modulus of the Bloch vector $v(t)$ may have rather sharp peaks associated with the evolution of initial correlations (see, e.g., Fig. 4).

Especially interesting is the ohmic case $(s=1)$ where the time dependence of the coherences is very sensitive to the value of the coupling constant $\lambda_{1}$. Figure 5 illustrates the kind of the behavior of the qubit entropy one might expect in this case. We call attention to the fact

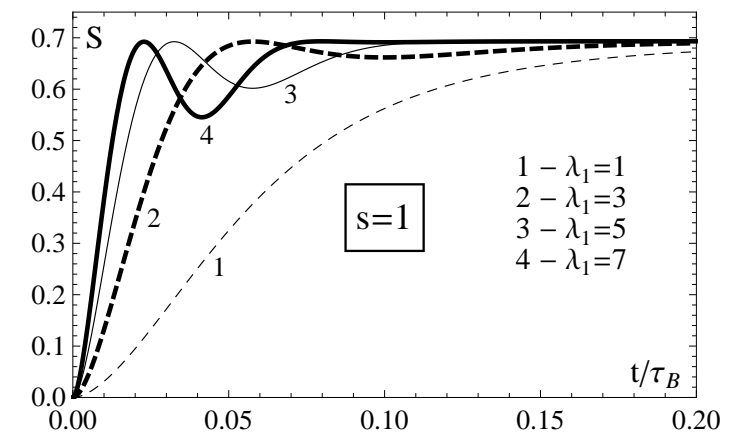

FIG. 5. Time evolution of the qubit entropy in the ohmic case for different coupling strengths. Parameter values: $\Omega \tau_{B}=10$, $\omega_{0} \beta=0.1$. that in some time intervals the entropy for a larger coupling constant is lower than that for a smaller coupling constant.

\section{CONCLUSIONS}

Let us present a summary of the results obtained in this work and discuss their relation to problems in open system dynamics.

We have derived exact formulas (27) and $(28)$ which describe the time behavior of the coherences $\left\langle\sigma_{ \pm}(t)\right\rangle$ (offdiagonal elements of the qubit density matrix) for all correlated initial states of the form (13) and (14), respectively. Initial states of these types can be interpreted physically in terms of the general theory of quantum measurements 1 , 19, 20]. In particular, operators $\Omega_{m}$ entering Eq. 27) represent operations during the "preparation" of a combined qubit-bath system in an initial state at temperature $T=1 / \beta$.

In this paper we have studied in detail the reduced qubit dynamics for the initial condition (14) which corresponds to the preparation of the qubit in some pure state $|\psi\rangle$. Since the qubit is not isolated from the bath, initial correlations come into play through the initial bath density matrix (18) which is a functional of $|\psi\rangle$ [see also Eq. 21p].

The quantity of most interest is the decoherence function $\widetilde{\gamma}(t)$ which determines the decay of the coherences: $\left\langle\sigma_{ \pm}(t)\right\rangle \propto \exp [-\widetilde{\gamma}(t)]$. Within the framework of the model under consideration, the decoherence function can be split into the vacuum part $\gamma_{\text {vac }}(t)$, the thermal part $\gamma_{\text {th }}(t)$, and the "correlation" part $\gamma_{\text {corr }}(t)$. In Sec. IV we have established some connections (with different levels of generality) between the properties of the bath spectral density $J(\omega)$ and possible regimes of decoherence. First, we have simple but quite general relations (36) and (38) which show that the long-time behavior of all the contributions to the total decoherence function depends crucially on the form of $J(\omega)$ in the low-frequency range. In particular, inequalities $\sqrt{36}$ give sufficient conditions for the regime of "incomplete decoherence" when $\left\langle\sigma_{ \pm}(t)\right\rangle$ do not tend to zero as $t \rightarrow \infty$. Second, assum- 
ing only that in the low-frequency range $J(\omega)$ scales as $J(\omega) \propto \omega^{s}$ with $s>0$, we have shown that the regime of "incomplete decoherence" occurs for $s>2$ when the total decoherence function is bounded. Finally, taking the bath spectral density in the form (41), we have derived exact expressions for all the terms in $\widetilde{\gamma}(t)$. As expected, these expressions confirm the earlier general predictions.

We have seen that the qualitative features of decoherence are essentially determined by the behavior of $J(\omega)$ in the range $\omega \ll \Omega$, where $\Omega$ is a cutoff frequency, characteristic of the bath modes. Thus, although explicit results have been obtained for a special (exponential) form of the cutoff function in $J(\omega)$, there is a good reason to think that the conclusions summarized in Table I apply as well to other forms of the cutoff function.

Decoherence is a fundamental property of open quantum systems which are met in quantum optics, electronics, atomic and molecular physics, etc. The literature concerning diverse aspects of this phenomenon is now quite voluminous. At first sight a study of very simple models like that specified by the Hamiltonian (1) might appear to be essentially a problem of applied mathematics. In a sense this is true. It should be noted, however, that the pure dephasing mechanism of decoherence described by the model (1) can dominate in real physical systems [21, 22. From this point of view exactly solvable dephasing models seem as themselves to deserve thorough studies. Note also that exact results obtained for a dephasing model can serve for constructing approximate solutions for more complicated dissipative systems [1] if the dissipative coupling with environment is weak. Another field of applications of exactly solvable dephasing models with initial system-environment correlations is the theory of quantum information. As a first step it would be desirable to generalize the results to many-qubit systems (quantum registers).

Recent works [10, 23] show that initial systemenvironment correlations can play an interesting and somewhat unexpected role in decoherence. We hope that the results of the present paper will be useful for further studies of correlations effects in decoherence phenomena.

\section{ACKNOWLEDGMENTS}

This work was supported by DFG (Deutsche Forschungsgemeinschaft), SFB 652 (Sonderforschungsbereich - Collective Research Center 652).

\section{Appendix A: Heisenberg equations of motion}

Since $\sigma_{3}$ commutes with the Hamiltonian (1), equations of motion for $b_{k}(t)$ and $b_{k}^{\dagger}(t)$ are

$i \frac{d b_{k}(t)}{d t}=\omega_{k} b_{k}(t)+\sigma_{3} g_{k}, \quad-i \frac{d b_{k}^{\dagger}(t)}{d t}=\omega_{k} b_{k}^{\dagger}(t)+\sigma_{3} g_{k}^{*}$. The solution of these equations with initial conditions $b_{k}(0)=b_{k}$ and $b_{k}^{\dagger}(0)=b_{k}^{\dagger}$ is easily found to be given by Eqs. (4). Equation of motion for $\sigma_{+}(t)$ reads:

$$
i \frac{d \sigma_{+}(t)}{d t}=-\omega_{0} \sigma_{+}(t)-2 \sum_{k}\left[g_{k} b_{k}^{\dagger}(t)+g_{k}^{*} b_{k}(t)\right] \sigma_{+}(t) .
$$

Using Eqs. (4) and relation $\sigma_{3} \sigma_{+}(t)=\sigma_{+}(t)$, we get

$$
i \frac{d \sigma_{+}(t)}{d t}=-\omega_{0} \sigma_{+}(t)-W(t) \sigma_{+}(t)
$$

with

$$
\begin{aligned}
W(t)=2 \sum_{k}\left\{g_{k} \mathrm{e}^{i \omega_{k} t}\left[b_{k}^{\dagger}+\alpha_{k}^{*}(t) / 2\right]\right. \\
\left.+g_{k}^{*} \mathrm{e}^{-i \omega_{k} t}\left[b_{k}+\alpha_{k}(t) / 2\right]\right\} .
\end{aligned}
$$

The solution of Eq. A1 is given by

$$
\sigma_{+}(t)=\exp \left(i \omega_{0} t\right) \exp _{+}\left[i \int_{0}^{t} W(\tau) d \tau\right] \sigma_{+}
$$

where $\exp _{+}[\ldots]$ is the chronologically ordered exponent. Expression A3 can be simplified using the operator identity

$$
\exp _{+}\left[i \int_{0}^{t} A(\tau) d \tau\right]=\exp \left\{-\frac{1}{2} \int_{0}^{t} d \tau_{1} \int_{0}^{\tau_{1}} d \tau_{2}\left[A\left(\tau_{1}\right), A\left(\tau_{2}\right)\right]\right\} \exp \left[i \int_{0}^{t} A(\tau) d \tau\right]
$$

which is valid if the commutator $\left[A\left(\tau_{1}\right), A\left(\tau_{2}\right)\right]$ at different times is a $c$-number function. It is easily verified that the operator $\mathrm{A} 2$ satisfies

$$
\left[W\left(t_{1}\right), W\left(t_{2}\right)\right]=-8 i \sum_{k}\left|g_{k}\right|^{2} \sin \left[\omega_{k}\left(t_{1}-t_{2}\right)\right]
$$

Applying Eq. A4 to $W$ and evaluating the integrals on the r.h.s. of that formula, one shows that the $c$-number functions cancel. Then from Eq. A3 follow expressions 6. for $\sigma_{+}(t)$ and $\sigma_{-}(t)=\sigma_{+}^{\dagger}(t)$. 


\section{Appendix B: Time derivative of $\gamma_{\mathbf{t h}}(t)$}

Let us consider the time derivative of $\gamma_{\mathrm{th}}(t)$ given by Eq. (35). We have

$$
\frac{d \gamma_{\mathrm{th}}}{d t}=2 \int_{0}^{\infty} d \omega \frac{J(\omega)}{\mathrm{e}^{\beta \omega}-1} \frac{\sin \omega t}{\omega}
$$

Under the assumption $(39)$, this reads

$$
\frac{d \gamma_{\mathrm{th}}}{d t}=\frac{2 \lambda_{s}(\Omega \beta)^{1-s}}{\beta} \int_{0}^{\infty} d x \frac{x^{s-1} F(x / \Omega \beta)}{\mathrm{e}^{x}-1} \sin (x t / \beta) .
$$

In the limit $\Omega \beta \gg 1$, the cutoff function may be replaced by $F(0)=1$. Then, performing the $x$-integral, we get

$$
\frac{d \gamma_{\mathrm{th}}}{d t}=\frac{2 \lambda_{s}(\Omega \beta)^{1-s}}{\beta} \Gamma(s) \sum_{k=1}^{\infty} \frac{\sin \left[s \tan ^{-1}(t / \beta k)\right]}{\left[k^{2}+(t / \beta)^{2}\right]^{s / 2}}
$$

where $\Gamma(s)$ is the gamma function. If $0<s \leq 2$, we see that $d \gamma_{\mathrm{th}} / d t>0$ for any $t>0$. However, this fact is not sufficient to conclude that the decoherence function diverges as $t \rightarrow \infty$ since it does not exclude the possibility that $d \gamma_{\mathrm{th}} / d t \rightarrow 0^{+}$as $t \rightarrow \infty$ and $\gamma_{\mathrm{th}}(t)$ saturates to a finite value. Relation $38 \mathrm{~b}$ ) excludes this possibility. It tells us that, for $0<s \leq 2$, the decoherence function $\gamma_{\text {th }}(t)$ cannot have a definite long-time limit since, if it had, the integral on the r.h.s. would be finite. We thus conclude that $\gamma_{\mathrm{th}}(t)$ is a monotonically increasing function and tends to infinity as $t \rightarrow \infty$.

If the bath spectral density is given by Eq. (41), the cutoff function in Eq. (B2) has the form $F(x / \Omega \beta)=$ $\exp (-x / \Omega \beta)$. Now the integration over $x$ leads to

$$
\begin{aligned}
& \frac{d \gamma_{\mathrm{th}}(t)}{d t}=\frac{2 \lambda_{s}(\Omega \beta)^{1-s}}{\beta} \Gamma(s) \\
& \quad \times \sum_{k=1}^{\infty} \frac{\sin \left[s \varphi_{k}(t)\right]}{\left[(k+1 / \Omega \beta)^{2}+(t / \beta)^{2}\right]^{s / 2}}, \quad(s>0),
\end{aligned}
$$

where $\varphi_{k}(t)$ is given by Eq. 45 . Since $0<\varphi_{k}(t)<\pi / 2$ for all $t>0$, we may employ the same arguments as above. Thus, for $0<s \leq 2$, the thermal term $\gamma_{\mathrm{th}}(t)$ monotonically increases with time and $\gamma_{t h}(t) \rightarrow \infty$ as $t \rightarrow \infty$. A more elaborate analysis of Eqs. (B3) and ( which will not be given here, shows that in the regime of "incomplete decoherence" the derivative $d \gamma_{\mathrm{th}}(t) / d t$ is a positive definite function of time for $2<s \leq 3$ and may change sign for $s>3$. In the latter case the thermal term $\gamma_{\mathrm{th}}(t)$ exhibits nonmonotonic time behavior.

\section{Appendix C: Exponential form of the density matrix}

The derivation of formula (54) is based on the following property of the Pauli matrices (see Problem 1 to $\S 55$ in Ref. [18]). Let $f$ be some function of the operator $a+\vec{\sigma} \cdot \vec{u}$, where $a$ and $\vec{u}$ are $c$-number quantities. Then

$$
f(a+\vec{\sigma} \cdot \vec{u})=A+\vec{\sigma} \cdot \vec{B},
$$

where

$$
\begin{aligned}
A & =\frac{1}{2}[f(a+u)+f(a-u)], \\
\vec{B} & =\frac{1}{2} \frac{\vec{u}}{u}[f(a+u)-f(a-u)],
\end{aligned}
$$

and $u=|\vec{u}|$. Identifying the r.h.s. of Eq. C1 with the qubit density matrix written in the form (53), we have $A=1 / 2$ and $\vec{B}=\vec{v} / 2$. On the l.h.s. of Eq. C1 we take $f(x)=\exp (x)$. Then Eqs. C2 give

$$
1=\mathrm{e}^{a}\left(\mathrm{e}^{u}+\mathrm{e}^{-u}\right), \quad v=\mathrm{e}^{a}\left(\mathrm{e}^{u}-\mathrm{e}^{-u}\right) .
$$

Solving these equations for $a$ and $u$, we find

$$
\mathrm{e}^{a}=\frac{1}{2} \sqrt{1-v^{2}}, \quad u=\frac{1}{2} \ln \left(\frac{1+v}{1-v}\right) .
$$

This leads immediately to the representation (54) for the density matrix.
[1] H.-P. Breuer and F. Petruccione, The Theory of Open Quantum Systems (Oxford University Press, Oxford, 2002).

[2] U. Weiss, Quantum Dissipative Systems (World Scientific, Singapore, 1999).

[3] S. Nakajima, Prog. Theor. Phys. 20, 948 (1958).

[4] R. Zwanzig, J. Chem. Phys. 33, 1338 (1960).

[5] B. Vacchini and H.-P. Breuer, Phys. Rev. A 81, 042103 (2010).

[6] A. Smirne and B. Vacchini, Phys. Rev. A 82, 022110 (2010).

[7] A. Steane, Rep. Prog. Phys. 61, 117 (1998).

[8] D. Bouwmeester, A. Ekert, and A. Zeilinger (eds.), The Physics of Quantum Information (Springer-Verlag,
Berlin, 2000).

[9] K.A. Valiev, Phys.-Usp. 48, 1 (2005).

[10] J. Dajka and J. Łuczka, Phys. Rev. A 82, 012341 (2010).

[11] J. Łuczka, Physica A 167, 919 (1990).

[12] W.G. Unruh, Phys. Rev. A 51, 992 (1995).

[13] G. M. Palma, K.-A. Suominen, and A.K. Ekert, Proc. R. Soc. London, Ser. A 452, 567 (1996).

[14] G. Doetsch, Introduction to the Theory and Application of the Laplace Transformation (Springer, Berlin, 1974).

[15] A.J. Leggett et al., Rev. Mod. Phys. 59, 1 (1987).

[16] L. Allen and J.H. Eberly, Optical Resonance and Twolevel Atoms (Dover, N.Y., 1975).

[17] C. Cohen-Tannoudji, B. Diu, and F. Laloë, Quantum Mechanics (Wiley, N.Y., 1977). 
[18] L.D. Landau and E.M. Lifshitz, Quantum Mechanics (Pergamon Press, Oxford, 1977).

[19] K. Kraus, States, Effects, and Operations Vol. 190 of Lecture Notes in Physics (Springer, Berlin, 1983).

[20] V.B. Braginsky and F.Ya. Khalili, Quantum Measurement (Cambridge University Press, Cambridge, 1992).
[21] D.I. Schuster et al., Nature (London) 445, 515 (2007).

[22] A.D. Cronin, J. Schmiedmayer, and D.E. Pritchard, Rev. Mod. Phys. 81, 1051 (2009).

[23] E.-M. Laine, J. Piilo, and H.-P. Breuer, EPL 92, 60010 (2010). 